\title{
Egalitarian property for power indices
}

\author{
Josep Freixas · Dorota Marciniak
}

Received: 3 November 2010 / Accepted: 25 August 2011 / Published online: 21 September 2011

C The Author(s) 2011. This article is published with open access at Springerlink.com

\begin{abstract}
In this study, we introduce and examine the Egalitarian property for some power indices on the class of simple games. This property means that after intersecting a game with a symmetric or anonymous game the difference between the values of two comparable players does not increase. We prove that the Shapley-Shubik index, the absolute Banzhaf index, and the Johnston score satisfy this property. We also give counterexamples for Holler, Deegan-Packel, normalized Banzhaf and Johnston indices. We prove that the Egalitarian property is a stronger condition for efficient power indices than the Lorentz domination.
\end{abstract}

\section{Introduction}

The main motivation of this study is to answer the following question: Does consensus increase egalitarianism in social choice? To answer this question we will deal with the analysis of power distribution for some power indices. For this purpose, we introduce and study the Egalitarian property for power indices.

The question of fairness and equality in the distribution of power is an important subject of interest, and it is a widely considered concept in the political, social,

\footnotetext{
J. Freixas

Department of Applied Mathematics III and High Engineering School of Manresa, Technical University of Catalonia, Barcelona, Spain

e-mail: josep.freixas@upc.edu

D. Marciniak ( $\varangle)$

National Institute of Telecommunications, Ul. Szachowa 1, 04-894 Warsaw, Poland e-mail: Dorota.address@gmail.com

D. Marciniak

Centre for Research and Studies in Sociology, CIES-ISCTE-IUL, Lisbon, Portugal
} 
and economical context (see for example Gambarelli and Owen 2002; Laruelle and Valenciano 2002; Holler 2002; Turnovec 2002; Felsenthal and Machover 2001).

Typically in the legislative bodies, a quorum is necessary for legislation to be passed. The procedure used to pass a law considered of great importance requires a strong support, not just in the sense of majority but also about other issues. Often two voting rules need to be satisfied (a double majority voting rule). For example, in the European Union, a double majority voting takes the form of Qualified Majority Voting. ${ }^{1}$ This voting rule is an example of the common decision making process, which can be expressed in the form of "voting by count and account". It is popular on the international level (e.g., European Union) as well as in the local communities, and companies (e.g., via the shareholders). This method represents a compromise between power and equality. The equality is represented by a consensus rule.

We consider power indices from both sides, either as a measure of influence or as a payoff, as described in Felsenthal and Machover (1998). Power indices are important mathematical tools broadly used in Social Science, Political Science, and Economics. The property we introduce might have applications in these domains, as it refers to the notion of equalizing the power distribution.

The notion of egalitarian property introduced here is related to the notion of Lorentz domination, which can be studied on the class of complete simple games. It was considered, for example, in Peleg (1992) and Weymark (1981). The notion of egalitarian property can be considered on the class of all simple games, and therefore it can be used in more situations.

In Peleg (1992), Peleg proved that Lorentz domination is satisfied for the Shapley-Shubik index. In the last section of this study, we show that our result for Shapley-Shubik index improves Peleg's result. Indeed, egalitarian property in this case implies Lorentz domination for an efficient power index which is, for example, the Shapley-Shubik index. We also show that Lorentz domination does not imply the egalitarian property for efficient power indices, which makes egalitarian property a stronger condition in this sense.

The article is organized as follows. First, we give basic definitions and set notations in the preliminary part. Then, we prove the egalitarian property for the class of semi-indices and for the Johnston score. In the next section, we study the relation between the egalitarian property and Lorentz domination, and we show that for efficient power indices the egalitarian property implies Lorentz domination. This is the case of the Shapley-Shubik index, and therefore it makes the egalitarian property a stronger condition than Lorentz domination. We show that other well-known power indices do not satisfy this property by constructing examples. For the reader's convenience, the examples and all the proofs are included in an appendix at the end of the article.

\footnotetext{
1 This voting system is to be applied to almost all policy areas starting in 2014 under the Treaty of Lisbon. Any decision taken under this scheme will require the support of at least $55 \%$ of the members of the Council of the European Union, who must also represent at least $65 \%$ of the EU's citizens. More information on voting systems in the EU can be found for example at http://europa.eu/.
} 


\section{Preliminaries}

In this section, we present some background knowledge that will be used in further considerations. We provide the definitions, notation, and terminology.

\subsection{Simple games}

Definition 1 Let $N$ be the set of players. A simple game $G$ is defined by a subset $\operatorname{Win}(G)$ of the set of parts of $N$, denoted by $\mathscr{P}(N)$. The set $\operatorname{Win}(G)$ is defined with respect to the following property: if $S \subseteq S^{\prime} \subseteq N$ and $S \in \operatorname{Win}(G)$ then $S^{\prime} \in \operatorname{Win}(G)$. It is also required that $\emptyset \notin \operatorname{Win}(G)$ and $N \in \operatorname{Win}(G)$. Each subset $S \subseteq N$ is referred to as a coalition. The set $N$ is called the grand coalition. The members of the set $\operatorname{Win}(G)$ are called winning coalitions. A coalition which is not winning is called losing. The set of losing coalitions will be denoted as $\operatorname{Lo}(G)$. The set of winning (losing) coalitions of player $i$ will be denoted by $\operatorname{Win}_{i}(G)\left(\operatorname{Lo}_{i}(G)\right)$, i.e., $\operatorname{Win}_{i}(G)=\{S \in \operatorname{Win}(G): i \in S\}$ $\left(\operatorname{Lo}_{i}(G)=\{S \in \operatorname{Lo}(G): i \in S\}\right)$.

The set of all simple games with $n$ voters will be denoted by $S_{n}$.

An equivalent definition of simple game is given by a characteristic function. A characteristic function of a simple game $G$ is any function $v_{G}: \mathscr{P}(N) \rightarrow\{0,1\}$ which satisfies the following conditions:

(a) $v_{G}$ is monotonic, i.e., it preserves the inclusion order of $\mathscr{P}(N)$ : if $S \subseteq S^{\prime} \subseteq N$ and $v(S)=1$ then $v\left(S^{\prime}\right)=1$,

(b) $v_{G}(\emptyset)=0$,

(c) $v_{G}(N)=1$.

For any given simple game $G$ it holds $\operatorname{Win}(G)=v_{G}^{-1}(1)$.

Definition 2 Let $G$ be a simple game. Then a coalition $S \in \operatorname{Win}(G)$ is $\operatorname{minimal}$ winning if none of its proper sub-coalitions is winning. The set of minimal winning coalitions will be denoted by $\operatorname{Win}^{\min }(G)$ or simply by Win ${ }^{\min }$.

A coalition of $G$ is maximal losing if it is losing and all of its proper supra-coalitions are winning. The set of maximal losing coalitions will be denoted by $\operatorname{Lo}^{\max }(G)$ or just Lomax.

Remark 1 The set of minimal winning coalitions determines the game uniquely. The same applies to the set of maximal losing coalitions.

\subsection{Voting by "count and account"}

Definition 3 (Weighted Voting Game) Let $N=\{1,2, \ldots, n\}$ be the set of players and let us denote the set of associated non-negative real weights by $\left\{w_{1}, w_{2}, \ldots, w_{n}\right\}$. Let $q$ be a positive real quota. We can set a simple game by the following rule

$$
S \text { is winning } \Longleftrightarrow \sum_{i \in S} w_{i} \geq q
$$


A simple game of this form is called weighted voting game and is denoted by $\left[q ; w_{1}, w_{2}, \ldots, w_{n}\right]$. Thereafter, we will use the convention $w_{1} \geq w_{2} \geq \cdots \geq w_{n}$.

The form $\left[q ; w_{1}, \ldots, w_{n}\right]$ of a simple game is often called a weighted representation. A simple game can have many weighted representations. If $n>3$ there are simple games that cannot be represented as a weighted voting game.

Two simple but important classes of weighted voting games are "voting by count" and "voting by account" games. By "voting by count" we refer to the weighted simple game in which a coalition is winning if it contains more than half of the players as a "majority of the count rule". By "voting by account" we refer to the weighted simple game in which a coalition is winning if the sum of weights of its members exceeds half of the sum of weights of all the players as the "majority of the account rule". "Voting by count and account" refers to the simple game in which a coalition is winning if it is winning with respect to both rules.

Remark 2 Let us remark that the class of Voting by count and account games contains all weighted voting games, but also other games which are complete games. The definition of complete game is recalled in the following subsection.

\subsection{Swings and desirability relation}

Let us recall the meaning of swing in a coalition as well as the desirability relation. Both notions play an important role in this article.

Definition 4 (Down-swing) Let $G$ be a simple game. A player $i$ has a down-swing in a coalition $S$ if $S \in \operatorname{Win}_{i}(G)$ and $S \backslash\{i\} \notin \operatorname{Win}(G)$. We will denote it as $i \downarrow S$. The set of coalitions in which player $i$ has a down-swing will be denoted by $\operatorname{Sw}_{i}^{\downarrow}(G)$ or simply by $\mathrm{Sw}_{i}^{\downarrow}$.

Definition 5 (Up-swing) Let $G$ be a simple game. A player $i$ has an up-swing in a coalition $S$ if $S \in \operatorname{Lo}(G)$ and $S \cup\{i\} \in \operatorname{Win}_{i}(G)$. We will denote it as $i \uparrow S$. The set of coalitions in which player $i$ has up-swing will be denoted by $\operatorname{Sw}_{i}^{\uparrow}(G)$ or simply by $\mathrm{Sw}_{i}^{\uparrow}$.

The following is a well-known result.

Remark 3 The number of up-swings of a player $i$ is equal to its number of downswings for any simple game.

In this study, we will use the term swing interchangeably with down-swing and we will denote the set $S w_{i}^{\downarrow}$ also as $\mathrm{Sw}_{i}$. If a player $i$ has a swing vote in a coalition $S$ we will use the terms $i$ is decisive in $S$ or $S$ is a swing coalition of $i$.

Here, we present a short list of properties of players in simple games. A player $i \in N$ in a simple game $G$ is:

(1) winner if $\{i\} \in \operatorname{Win}(G)$,

(2) veto player if $i \in S$ for all $S \in \operatorname{Win}(G)$, 
(3) dictator if $\{i\}$ is the unique minimal winning coalition,

(4) null player if $i \notin S$ for all $S \in \operatorname{Win}^{\min }(G)$.

A player is a dictator if and only if it is a winner and has veto.

Definition 6 Let $G$ be a simple game,

$i \succsim_{D} j \quad$ iff $\quad S \cup\{j\} \in \operatorname{Win}_{j}(G) \Rightarrow S \cup\{i\} \in \operatorname{Win}_{i}(G) \quad$ for all $S \subseteq N \backslash\{i, j\}$

It is not difficult to check that $\succsim_{D}$ is a preordering. It is called desirability (resp., strict desirability) relation and $\sim_{D}$ is the equi-desirability relation (Isbell 1958).

When the desirability relation in a game $G$ should be distinguished from the desirability relation of another game $G^{\prime}$ we will use the notation $D_{[G]}$.

Definition 7 A simple game is complete if the desirability relation is total, i.e., for any two players $i$ and $j$ at least one of the following holds: $i \succsim_{D} j$ or $j{ }_{D} i$.

\subsection{Power indices}

Definition 8 A power index $\psi$ is a map $\psi: S_{n} \rightarrow \mathbb{R}_{+}^{n}$ that assigns to each simple game $v \in S_{n}$ a vector of $\mathbb{R}_{+}^{n}$, whose components represent the payoff or influence measure of each voter. The components of this function will be denoted by $\psi_{i}[v]$.

If we consider a normalized power index, we can replace $\mathbb{R}_{+}^{n}$ by $[0,1]^{n}$ in the codomain of $\psi$.

Let us recall briefly some well-known power indices for simple games. For references on them see among others: Banzhaf (1965), Shapley and Shubik (1954), Johnston (1978), Holler (1982), and Deegan and Packel (1978).

\section{Shapley-Shubik index}

$$
\mathrm{Sh}_{i}[G]=\frac{1}{|N| !} \sum_{S \in \mathrm{Sw}_{i}}(|S|-1) !(|N|-|S|) !
$$

\section{Banzhaf score}

$$
\beta_{i}^{\#}[G]=\left|\mathrm{Sw}_{i}(G)\right|
$$

Banzhaf normalized index

$$
\dot{\beta}_{i}[G]=\frac{\beta_{i}[G]}{\sum_{j \in N} \beta_{j}[G]}
$$

\section{Banzhaf absolute index}

$$
\beta_{i}[G]=\beta^{\#}[G] / 2^{|N|-1}
$$


The Banzhaf normalized index is also called the "relative Banzhaf index" to distinguish it from its other versions. Banzhaf index is also known as Penrose-BanzhafColeman index.

\section{Johnston score}

$$
\mathscr{S}_{i}^{\#}[G]=\sum_{S \in \mathrm{Sw}_{i}} \frac{1}{d_{S}}
$$

where $d_{S}=\sum_{i \in S} d_{i}(S)$ and $d_{i}(S)=v_{G}(S)-v_{G}(S \backslash\{i\})$ is the marginal contribution of player $i$ into $S$. Then $d_{S}$ is equal to the number of decisive members of $S$.

Johnston index

$$
\dot{\mathscr{S}}_{i}[G]=\frac{\mathscr{S}_{i}[G]}{\sum_{j \in N} \mathscr{S}_{j}[G]}
$$

\section{Holler index}

$$
\dot{\chi}_{i}[G]=\frac{\chi_{i}^{\#}[G]}{\sum_{j \in N} \chi_{j}[G]}
$$

where

$$
\chi_{i}^{\#}[G]=\left|\operatorname{Win}_{i}^{\min }[G]\right|
$$

We will refer to $\chi^{\#}$ as Holler score.

\section{Deegan-Packel index}

$$
\dot{\delta}_{i}[G]=\frac{1}{\left|\operatorname{Win}^{\min }\right|} \sum_{S \in \operatorname{Win}_{i}^{\min }[G]} \frac{1}{|S|}
$$

We will refer to $\sum_{S \in \operatorname{Win}_{i}^{\text {min }}[G]} \frac{1}{|S|}$ as Deegan-Packel score of player $i$ in the game $G$ and we will denote it by $\delta_{i}^{\#}[G]$.

Definition 9 A semi-index $\psi$, or semivalue for simple games, is a power index on the class of simple games that satisfies:

(1) symmetry: for any simple game $v$, and for any bijection $\theta: N \rightarrow N$, it is $\psi_{\theta(i)}[\theta v]=\psi_{i}[v]$,

where the simple game $\theta v$ is defined by $\theta v(S)=v(\theta(S))$ for any coalition $S$.

(2) positivity: $\psi[v] \geq 0$, for all $v \in S_{n}$.

(3) dummy player property: if $i$ is a dummy player in game $v$, i.e., $v(S \cup\{i\})=$ $v(S)+v(\{i\})$ for all $S \subseteq N \backslash\{i\}$, then $\psi_{i}[v]=v(\{i\})$. 
(4) transfer property: for any two simple games $u$ and $v$

$$
\psi[u \vee v]=\psi[u]+\psi[v]-\psi[u \wedge v]
$$

where $(u \vee v)(S)=\max \{u(S), v(S)\}$ and $(u \wedge v)(S)=\min \{u(S), v(S)\}$.

The class of semi-indices is a broad and important class of power indices. A good survey on semi-indices is Carreras et al. (2003). Let us recall that the Shapley-Shubik index and the Banzhaf absolute index are semi-indices. In Freixas (2010), the ordinal equivalence of these two semi-indices it is studied and several subclasses of semiindices are also considered: regular, binomial, or segment semi-indices. However, the indices defined in: (2), (3), (5), (6), (7), and (8) are not semi-indices. In this article, we deal with all the cases: the class of semi-indices and the remaining six indices just mentioned above. In the next section, we define the egalitarian property and prove that it is satisfied for the class of semi-indices and also for the Johnston score.

\section{The egalitarian property for power indices}

The property we introduce here is related to a power analysis in simple games with different levels of consensus. One real-life example of two voting systems with two different levels of consensus are the decision rules adopted in the Nice European summit, held in December 2000, for the European Union enlargement voting system:

Example 1 In the European Union Council there are the following players (in this context countries), which are listed with respect to populations:

[1] Germany, [2] United Kingdom, [3] France, [4] Italy, [5] Spain, [6] Poland, [7] Romania, [8] The Netherlands, [9] Greece, [10] Czech Republic, [11] Belgium, [12] Hungary, [13] Portugal, [14] Sweden, [15] Bulgaria, [16] Austria, [17] Slovak Republic, [18] Denmark,

[19] Finland, [20] Ireland, [21] Lithuania, [22] Latvia, [23] Slovenia, [24] Estonia, [25] Cyprus, [26] Luxembourg, [27] Malta.

and two rules of voting:

$$
v_{1} \cap v_{2} \cap v_{3} \quad v_{1} \cap v_{2}^{\prime} \cap v_{3}
$$

where

$$
\begin{aligned}
v_{1}= & {[\mathbf{2 5 5} ; 29(4), 27(2), 14,13,12(5), 10(3), 7(5), 4(5), 3] } \\
v_{2}=[\mathbf{1 4} ; & 1(25)] \\
v_{2}^{\prime}=[\mathbf{1 8} ; & 1(25)] \\
v_{3}=[\mathbf{6 2 0} ; & 170,123,122,120,82,80,47,33,22,21(4) \\
& 18,17(2), 11(3), 8(2), 5,4,3,2,1(2)]
\end{aligned}
$$

The notation 29(4) in $v_{1}$ means that the four biggest countries: 1-Germany, 2-Great Britain, 3-France and 4-Italy have weight 29 in $v_{1}$, and so on. For example, Poland is the sixth most populated country and therefore has weight 27 in $v_{1}$, weight 1 in $v_{2}$ and in $v_{2}^{\prime}$, and weight 80 in $v_{3}$. 
Comparing the two decision rules, we see that they can be represented as an intersection of a simple game $G=v_{1} \cap v_{3}$ and a symmetric game ( $v_{2}$ or $v_{2}^{\prime}$ ). In the first rule, the quorum is 14 , while in the second rule it is 18 . In this section, we study how does the voting power distribution change when we increase the quorum, i.e., the level of consensus.

\subsection{Level of consensus in a simple game}

Let us establish some basic definitions and notations used in further considerations.

A symmetric game is a simple weighted voting game of the form $[\gamma ; \underbrace{1,1, \ldots, 1}_{n}]$.

It is denoted by $Q_{\gamma}$ and we will refer to $\gamma$ as the quorum number (or consensus level) for $Q_{\gamma}$, or simply quorum.

In a symmetric game all the participants are equivalent by the desirability relation. In the simple game $G_{\gamma}=G \cap Q_{\gamma}$ two comparable participants that are not equi-desirable in $G$ can be equalized by the desirability relation in $G_{\gamma}$. Therefore, we can expect that in the game $G_{\gamma}$, which appears to be more egalitarian than $G$ with regard to the desirability relation, actors will be treated more equally. This accords with the intuition as we pass from the generic simple game $G$ to $G_{\gamma}$ only requiring one additional consensus condition.

Definition 10 We say that a simple game $G_{2}$ has a higher level of consensus than $G_{1}$ if these games can be represented as $G_{1}=G \cap Q_{\gamma_{1}}$ and $G_{2}=G \cap Q_{\gamma_{2}}$ with $\gamma_{2}>\gamma_{1}$.

Note that it might be $G_{\gamma_{1}}=G_{\gamma_{2}}$ even if $\lambda_{2}>\lambda_{1}$. A very simple example of such possibility is obtained by taking $G$ as the symmetric game $Q_{\lambda}$ with $\lambda \geq \lambda_{2}$.

As remarked before, the desirability relation in $G_{\gamma}$ can equalize players that were not equivalent in $G$. However, the order obtained in $G_{\gamma}$ is not contradictory to the desirability relation of $G$, i.e., it never happens that $i \succ_{D[G]} j$ and $j \succ_{D\left[G_{\gamma}\right]} i$ for arbitrary players $i$ and $j$.

Let us remark that if two players are comparable in $u \cap Q_{\gamma}$ then they remain comparable in all games $u \cap Q_{\gamma^{\prime}}, \gamma^{\prime} \geq \gamma$. Moreover, if $i \succ_{D\left[u \cap Q_{\gamma}\right]} j$ then $i \succsim_{D\left[u \cap Q_{\gamma^{\prime}}\right]} j$ for all $\gamma^{\prime} \geq \gamma$ and if $i \sim_{D\left[u \cap Q_{\gamma}\right]} j$ then $i \sim_{D\left[u \cap Q_{\gamma^{\prime}}\right]} j$ for all $\gamma^{\prime} \geq \gamma$.

When two players in a game $G$ are equalized in $G_{\gamma}$ with respect to the desirability relation then they become equally powerful. Consequently, we expect an arbitrary power index to preassign the same value to both players. Evidently, for players that are equivalent in $G_{\gamma}$ the differences of evaluations given by the power index are not increased when passing from $G$ to $G_{\gamma}$. We shall require this behavior not only for players being symmetric in $G_{\gamma}$ but also for all players. This property of power indices will be called the egalitarian property.

Definition 11 Let $G$ be a simple game. Consider an egalitarian sequence of games:

$$
\left(G \cap Q_{\gamma}\right)_{\gamma=1,2, \ldots, n}
$$

where $Q_{\gamma}$ is a symmetric game at consensus level $\gamma$. We say that an index $\psi$ has the egalitarian property if for each simple game $G$ and for any two comparable players 
$i$ and $j(i \succsim D j)$ :

$$
\psi_{i}\left[G_{\gamma}\right]-\psi_{j}\left[G_{\gamma}\right] \geq \psi_{i}\left[G_{\gamma+1}\right]-\psi_{j}\left[G_{\gamma+1}\right]
$$

where $\psi_{i}\left[G_{\gamma}\right]$ is the value of player $i$ for index $\psi$ in game $G_{\gamma}=G \cap Q_{\gamma}$.

If a power index satisfies the egalitarian property, then the index is more scattered in a game with a prefixed consensus than in a game with a higher level of consensus. For example, if the power index is considered as a payoff distribution, the egalitarian property means that the higher the level of consensus the smaller the difference of payoffs of any pair of voters.

\subsection{Egalitarian property for the class of semi-indices}

Theorem 1 Every semi-index satisfies the egalitarian property.

Corollary 1 The Banzhaf absolute index and the Shapley-Shubik index satisfy the egalitarian property.

Remark 4 All regular semi-indices (semi-indices with positive coefficients) preserve the desirability relation, see Carreras and Freixas (2008), but the non-regular semi-indices do not necessarily preserve it. However, all semi-indices do satisfy the egalitarian property because the order given by the desirability relation never reverses the order given by semi-indices.

\subsection{The Egalitarian property for the Johnston score}

Theorem 2 The Johnston score $\mathscr{J}$ satisfies the egalitarian property.

\subsection{Indices that do not satisfy the egalitarian property}

It is important to remark that if a power index preserves the desirability relation it does not imply that the power index satisfies the egalitarian property. The following result provides remarkable examples of indices that fit in this category:

Remark 5 The normalized versions of the Banzhaf and Johnston indices do not satisfy the egalitarian property.

Other known power indices not preserving the desirability relation also do not satisfy the egalitarian property.

Remark 6 The both versions, the raw (the score) and the normalized, of the Holler and the Deegan-Packel indices do not satisfy the egalitarian property.

As a corollary of this remark, the egalitarian property is not satisfied by other indices. For example, the Shift-power index (see Alonso-Meijide and Freixas 2010), which also does not preserve the desirability relation, does not satisfy the egalitarian property. 


\section{Egalitarian property and Lorentz domination}

In this section, we show that our result concerning the Shapley-Shubik index improves the result of Peleg (1992, Theorem 3.1) (see also Weymark 1981) on Lorentz domination for complete simple games. Peleg's result states that for a complete simple game $u$ and games $v_{1}=u \cap Q_{\gamma_{1}}$ and $v_{2}=u \cap Q_{\gamma_{2}}$ with $0 \leq \gamma_{1}<\gamma_{2} \leq 1, \operatorname{Sh}\left(v_{2}\right)$ Lorentz-dominates $\operatorname{Sh}\left(v_{1}\right)$, i.e.,

$$
\sum_{i=j}^{n} \operatorname{Sh}_{i}\left[v_{2}\right] \geq \sum_{i=j}^{n} \operatorname{Sh}_{i}\left[v_{1}\right] \text { for all } j=1,2, \ldots, n
$$

Thereafter, we use the convention that the players are enumerated with decreasing desirability order, i.e., $1 \succsim_{D[u]} 2 \succsim_{D[u]} \cdots \succsim_{D[u]} n$ for all $u \in S_{n}$. In that situation Carreras and Freixas (2004) note that Peleg's result also holds for simple games $u$ as long as $v_{1}$ becomes a complete simple game. From efficiency and the Lorentzdomination of the Shapley-Shubik index we get:

(i) $\operatorname{Sh}_{1}\left[v_{1}\right] \geq \operatorname{Sh}_{1}\left[v_{2}\right]$ and

(ii) $\mathrm{Sh}_{n}\left[v_{1}\right] \geq \mathrm{Sh}_{n}\left[v_{2}\right]$.

This result reflects the egalitarianism of the Shapley-Shubik index (see Carreras and Freixas 2004) in the sense that:

$$
\mathrm{Sh}_{1}\left[v_{1}\right]-\mathrm{Sh}_{n}\left[v_{1}\right] \geq \operatorname{Sh}_{1}\left[v_{2}\right]-\operatorname{Sh}_{n}\left[v_{2}\right]
$$

Our result concerning egalitarian property for the Shapley-Shubik index (Theorem 1) improves Peleg's result in at least two aspects:

(1) We consider the class of all simple games (not only the class of complete simple games), as we investigate pairs of players comparable by the desirability relation.

Theorem 1 can be applied to non-comparable players $i$ and $j$ in a simple game $u$, provided that they become comparable in $u \cap Q_{\gamma}$ for some $\gamma$. Thus, Theorem 1 can be applied to compare two individual players in a non-complete game, when the assumptions of Peleg's theorem do not hold.

(2) Equation 10 does not only hold for the extreme players (the strongest and the weakest) but for any pair of comparable players.

Theorem 1 does not follow from the Lorentz domination even if we additionally assume efficiency. As an example see counterexample 4, where the Lorentz domination holds for Banzhaf and Johnston indices, but not the egalitarian property. However,

(3) Egalitarian property and efficiency imply Lorentz domination, as we will prove in Theorem 3.

Thus, our Theorem 3 is an independent and stronger result.

Theorem 3 Egalitarian property and efficiency of a power index, which preserves the desirability order imply its Lorentz domination in the class of complete simple games. 
Therefore, even in the class of complete simple games for an efficient index $\psi$ which respects the desirability order we have the following:

$$
\text { Egalitarian property } \underset{\nLeftarrow}{\Rightarrow} \text { Lorentz domination }
$$

\section{Conclusions}

The results shown in this article give an explicit answer to the question of equalizing the power distribution by adding a quorum condition to the game. We can deduce that not all the known power indices reflect in required way egalitarianism among the players.

Among the considered indices only the Shapley-Shubik index satisfies the egalitarian property being efficient at the same time. This can be regarded as a counter-intuitive behavior of the rest of considered indices. We conclude that it is better to use non normalized versions of Banzhaf and Johnston indices if are interested in decreasing the differences of values between pairs of players. The results obtained here can have some impact in topics like economic distance or social inequalities.

An important application of the results of this article can refer to a common voting system: voting by count and account. It is a double majority rule composed of the simple majority rule (voting by count) and the weighted majority rule (voting by account). This decision making system has a long tradition and it is presently used in many electoral systems. Examples can be found, for instance, in Taylor and Zwicker (1999, p. 19), Taylor (1995), and Hirokawa and Xu (2005). It is of the interest to study this voting system as it appears in many real-life examples.

In Peleg (1992), it is proved that voting by count and account is more egalitarian for the Shapley-Shubik index than voting by account. This result answers the question, for the case of Shapley-Shubik index, raised by Thomson in a letter to Aumann: to find a relationship between game theoretic solutions of voting by account versus by count and account. However, he left opened the question for other solutions.

In a recent article, Hirokawa and Vlach (2006), the authors address a similar question for the case of Banzhaf, Johnston, and Deegan-Packel scores. They show that an analogous shift of power occurs when the power distribution is measured by the Banzhaf and Johnston scores. On the other hand, they show that this is not true for the case of Deegan-Packel score. These results refer to the comparison of power distributions in voting by account and in voting by count and account. It is known that while both rules: the "count rule" and the "account rule" can be represented by weighted simple games their intersection does not necessarily admit a weighted representation (see Peleg 1992). However, the intersection of two weighted voting games is always a complete game (Definition 7) whenever their desirability orders are not contradictory, which is satisfied for the count and account rule. In this context, a subclass of complete games is the broadest class to study the question of increasing egalitarianism in a "count and account" decision making process.

In this article, we answer a more general question. We show that when replacing an "account" rule by a more general complete game we get a more egalitarian power distribution for the Shapley-Shubik index and Banzhaf and Johnston scores. We prove this also for the class of all semi-indices. These results generalize the results in Peleg 
(1992) and Hirokawa and Vlach (2006), in two directions: for a larger class of power indices, and for a larger class of games.

A further study to be done would be to study other power indices with respect to the egalitarian property, for example, other indices presented in the Voting Power and Power Index Website, http://powerslave.val.utu.fi/. Some results can yet be derived from the results of this study, as corollaries, for example, that the Coleman Collectivity Index (Coleman 1971), the Coleman Preventive Power Index (Coleman 1971), and Rae Index (Rae 1969) also satisfy the egalitarian property.

Acknowledgments Research partially funded by Grants SGR 2009-1029 of Generalitat de Catalunya and MTM 2009-08037 from the Spanish Science and Innovation Ministry, and by the Polish National Budget Funds 2010-2013 for Science under the Grant N N514 044438. The first author acknowledges the support of the Barcelona Graduate School of Economics and of the Government of Catalonia.

Open Access This article is distributed under the terms of the Creative Commons Attribution Noncommercial License which permits any noncommercial use, distribution, and reproduction in any medium, provided the original author(s) and source are credited.

\section{Appendices}

\section{A Proof of Theorem 1}

Every semi-index can be expressed as $\psi_{i}[G]=\sum_{k=1}^{n} \alpha^{k} \beta_{i}^{k}[G]$, where $\beta_{i}^{k}$ is defined

$$
\beta_{i}^{k}[G]=\left|\left\{S \in \mathrm{Sw}_{i}(G):|S|=k\right\}\right|
$$

and $\alpha^{k} \in \mathbb{R}_{+}\left(\alpha^{k}>0\right)$. Let us remark that $\alpha^{k}$ does not depend on the game $G$, and we have $\beta_{i}^{k}\left[G_{\gamma}\right]=0$ for $k<\gamma$. Thus, the sum can be represented as

$$
\psi_{i}\left[G_{\gamma}\right]=\alpha^{\gamma} \beta_{i}^{\gamma}\left[G_{\gamma}\right]+\alpha^{\gamma+1} \beta_{i}^{\gamma+1}\left[G_{\gamma}\right]+\sum_{k \geq \gamma+2} \alpha^{k} \beta_{i}^{k}\left[G_{\gamma}\right]
$$

Then the inequality (9) takes the form:

$$
\begin{aligned}
& \left(\alpha^{\gamma} \beta_{i}^{\gamma}\left[G_{\gamma}\right]+\alpha^{\gamma+1} \beta_{i}^{\gamma+1}\left[G_{\gamma}\right]+\sum_{k \geq \gamma+2} \alpha^{k} \beta_{i}^{k}\left[G_{\gamma}\right]\right) \\
& \quad-\left(\alpha^{\gamma} \beta_{j}^{\gamma}\left[G_{\gamma}\right]+\alpha^{\gamma+1} \beta_{j}^{\gamma+1}\left[G_{\gamma}\right]+\sum_{k \geq \gamma+2} \alpha^{k} \beta_{j}^{k}\left[G_{\gamma}\right]\right) \\
& \geq\left(\alpha^{\gamma+1} \beta_{i}^{\gamma+1}\left[G_{\gamma+1}\right]+\sum_{k \geq \gamma+2} \alpha^{k} \beta_{i}^{k}\left[G_{\gamma+1}\right]\right) \\
& \quad-\left(\alpha^{\gamma+1} \beta_{j}^{\gamma+1}\left[G_{\gamma+1}\right]+\sum_{k \geq \gamma+2} \alpha^{k} \beta_{j}^{k}\left[G_{\gamma+1}\right]\right)
\end{aligned}
$$

Every semi-index has the property that for $k>\gamma+1$

$$
\alpha^{k} \cdot \beta_{i}^{k}\left[G_{\gamma}\right]=\alpha^{k} \cdot \beta_{i}^{k}\left[G_{\gamma+1}\right]
$$


and so it is enough to prove that when we increase the quorum from $\gamma$ to $\gamma+1$ then

$$
\begin{gathered}
\alpha^{\gamma} \beta_{i}^{\gamma}\left[G_{\gamma}\right]-\alpha^{\gamma} \beta_{j}^{\gamma}\left[G_{\gamma}\right] \geq \alpha^{\gamma} \beta_{i}^{\gamma}\left[G_{\gamma+1}\right]-\alpha^{\gamma} \beta_{j}^{\gamma}\left[G_{\gamma+1}\right], \\
\alpha^{\gamma+1} \beta_{i}^{\gamma+1}\left[G_{\gamma}\right]-\alpha^{\gamma+1} \beta_{j}^{\gamma+1}\left[G_{\gamma}\right] \geq \alpha^{\gamma+1} \beta_{i}^{\gamma+1}\left[G_{\gamma+1}\right]-\alpha^{\gamma+1} \beta_{j}^{\gamma+1}\left[G_{\gamma+1}\right] .
\end{gathered}
$$

Note that the right hand side of the Eq. 11 is equal to 0, because both components on the right hand side are 0 .

To show the inequalities (11) and (12), since $\alpha^{\gamma}$ and $\alpha^{\gamma+1}$ are non-negative real numbers, it is enough to prove the following inequalities:

$$
\begin{gathered}
\beta_{i}^{\gamma}\left[G_{\gamma}\right]-\beta_{j}^{\gamma}\left[G_{\gamma}\right] \geq 0, \\
\beta_{i}^{\gamma+1}\left[G_{\gamma}\right]-\beta_{j}^{\gamma+1}\left[G_{\gamma}\right] \geq \beta_{i}^{\gamma+1}\left[G_{\gamma+1}\right]-\beta_{j}^{\gamma+1}\left[G_{\gamma+1}\right] .
\end{gathered}
$$

Note that (13) holds since $i \succsim D[G] j$. It is so for $k=\gamma$, because $\beta_{i}^{\gamma}\left[G_{\gamma}\right] \geq \beta_{j}^{\gamma}\left[G_{\gamma}\right]$ and $\beta_{i}^{\gamma}\left[G_{\gamma+1}\right]=0=\beta_{j}^{\gamma}\left[G_{\gamma+1}\right]$ as there is no winning coalition of cardinality $\gamma$ in the game $G_{\gamma+1}$. Let us now prove the Eq. 14 for $k=\gamma+1$. We will consider the following sets:

$$
\begin{aligned}
& S_{i, j}^{+}=\{S \subseteq N:|S|=\gamma+1, i, j \in S\} \\
& S_{i, j}^{-}=\{S \subseteq N:|S|=\gamma, i, j \notin S\}
\end{aligned}
$$

and let us consider the sets of up-swings $S w_{i}^{\uparrow}$ in $S_{i, j}^{-}$and down-swings $S w_{i}^{\downarrow}$ in $S_{i, j}^{+}$. Let us recall the definition of the sets of up-swings and down-swings:

$$
\begin{aligned}
& \operatorname{Sw}_{i}^{\uparrow}=\{S \subseteq N: i \notin S, S \notin \operatorname{Win}(G), S \cup\{i\} \in \operatorname{Win}(G)\} \\
& \operatorname{Sw}_{i}^{\downarrow}=\left\{S \in \operatorname{Win}_{i}(G): S \backslash\{i\} \notin \operatorname{Win}_{i}(G)\right\}
\end{aligned}
$$

In the case of $S_{i, j}^{+}$we have the following partition of this set:

$$
\begin{aligned}
& S_{1}^{+}=\{S \in \operatorname{Lo}(G):|S|=\gamma+1, i, j \in S\}=\operatorname{Lo}(G) \cap S_{i, j}^{+} \\
& S_{2}^{+}=\left\{S \in \operatorname{Sw}_{j}^{\downarrow}(G):|S|=\gamma+1, i, j \in S\right\}=\operatorname{Sw}_{j}^{\downarrow} \cap S_{i, j}^{+} \\
& S_{3}^{+}=\left\{S \in \operatorname{Sw}_{i}^{\downarrow}(G) \backslash \operatorname{Sw}_{j}^{\downarrow}(G):|S|=\gamma+1, i, j \in S\right\}=\left(\operatorname{Sw}_{i}^{\downarrow}(G) \backslash \operatorname{Sw}_{j}^{\downarrow}(G)\right) \cap S_{i, j}^{+} \\
& S_{4}^{+}=\left\{S \in \operatorname{Win}(G) \backslash \operatorname{Sw}_{i}^{\downarrow}:|S|=\gamma+1, i, j \in S\right\}=\left(\operatorname{Win}(G) \backslash \operatorname{Sw}_{i}^{\downarrow}\right) \cap S_{i, j}^{+}
\end{aligned}
$$


and we have the partition of $S_{i, j}^{-}$:

$$
\begin{aligned}
& S_{1}^{-}=\left\{S \in \operatorname{Lo}(G) \backslash \mathrm{Sw}_{i}^{\uparrow}:|S|=\gamma, i, j \notin S\right\}=\left(\operatorname{Lo}(G) \backslash \mathrm{Sw}_{i}^{\uparrow}\right) \cap S_{i, j}^{-} \\
& S_{2}^{-}=\left\{S \in \operatorname{Sw}_{i}^{\uparrow} \backslash \operatorname{Sw}_{j}^{\uparrow}(G):|S|=\gamma, i, j \notin S\right\}=\left(\mathrm{Sw}_{i}^{\uparrow} \backslash \mathrm{Sw}_{j}^{\uparrow}\right) \cap S_{i, j}^{-} \\
& S_{3}^{-}=\left\{S \in \operatorname{Sw}_{j}^{\uparrow}(G):|S|=\gamma, i, j \notin S\right\}=\operatorname{Sw}_{j}^{\uparrow}(G) \cap S_{i, j}^{-} \\
& S_{4}^{-}=\{S \in \operatorname{Win}(G):|S|=\gamma, i, j \notin S\}=\operatorname{Win}(G) \cap S_{i, j}^{-}
\end{aligned}
$$

Then in the games $G_{\gamma}$ and $G_{\gamma+1}$ :

$$
\begin{aligned}
\beta_{i}^{\gamma+1}\left[G_{\gamma}\right] & =\left|S_{2}^{+}\right|+\left|S_{3}^{+}\right|+\left|S_{2}^{-}\right|+\left|S_{3}^{-}\right| \\
\beta_{j}^{\gamma+1}\left[G_{\gamma}\right] & =\left|S_{2}^{+}\right|+\left|S_{3}^{-}\right| \\
\beta_{i}^{\gamma+1}\left[G_{\gamma+1}\right] & =\left|S_{2}^{+}\right|+\left|S_{3}^{+}\right|+\left|S_{2}^{-}\right|+\left|S_{3}^{-}\right|+\left|S_{4}^{+}\right|+\left|S_{4}^{-}\right| \\
& =\beta_{i}^{\gamma+1}\left[G_{\gamma}\right]+\left|S_{4}^{+}\right|+\left|S_{4}^{-}\right| \\
\beta_{j}^{\gamma+1}\left[G_{\gamma+1}\right] & =\left|S_{2}^{+}\right|+\left|S_{3}^{-}\right|+\left|S_{3}^{+}\right|+A S_{4}^{+}|+| S_{4}^{-} \mid \\
& =\beta_{j}^{\gamma+1}\left[G_{\gamma}\right]+\left|S_{3}^{+}\right|+\left|S_{4}^{+}\right|+\left|S_{4}^{-}\right|
\end{aligned}
$$

Then we get the inequality (14) by putting the above formulas into (14) and doing straightforward calculations $\left(\left|S_{3}^{+}\right| \geq 0\right)$. When $\left|S_{3}^{+}\right|>0$ then we get strict inequality in (14) and thus in (9), also if $\beta_{i}^{\gamma}\left[G_{\gamma}\right]>\beta_{j}^{\gamma}\left[G_{\gamma}\right]$ then we get strict inequality in (9).

\section{B Proof of Theorem 2}

The Johnston index can be expressed as $\mathscr{J}_{i}[G]=\sum_{k=1}^{n} \mathscr{J}_{i}^{k}[G]$, where we define $\mathscr{J}_{i}^{k}[G]=\sum_{S \in \mathrm{Sw}_{i}:|S|=k} 1 / d_{S}$, where $d_{S}$ is the number of decisive players in the coalition $S$. We calculate $d_{S}$ only for coalitions that have at least one decisive player thus $\frac{1}{d_{S}}$ is well defined. Moreover, $\mathscr{J}_{i}{ }^{k}\left[G_{\gamma}\right]=0$ for $k<\gamma$ thus the above sum can be represented as

$$
\mathscr{J}_{i}\left[G_{\gamma}\right]=\mathscr{J}_{i}^{\gamma}\left[G_{\gamma}\right]+\mathscr{J}_{i}^{\gamma+1}\left[G_{\gamma}\right]+\sum_{k \geq \gamma+2} \mathscr{J}_{i}^{k}\left[G_{\gamma}\right]
$$

Then the inequality (9), which we are going to prove, takes the form:

$$
\begin{aligned}
& \left(\mathscr{J}_{i}^{\gamma}\left[G_{\gamma}\right]+\mathscr{J}_{i}^{\gamma+1}\left[G_{\gamma}\right]+\sum_{k \geq \gamma+2} \mathscr{J}_{i}^{k}\left[G_{\gamma}\right]\right)-\left(\mathscr{J}_{j}^{\gamma}\left[G_{\gamma}\right]+\mathscr{J}_{j}^{\gamma+1}\left[G_{\gamma}\right]+\sum_{k \geq \gamma+2} \mathscr{J}_{j}^{k}\left[G_{\gamma}\right]\right) \\
& \leq\left(\mathscr{J}_{i}^{\gamma+1}\left[G_{\gamma+1}\right]+\sum_{k \geq \gamma+2} \mathscr{J}_{i}^{k}\left[G_{\gamma+1}\right]\right)-\left(\mathscr{J}_{j}^{\gamma+1}\left[G_{\gamma+1}\right]+\sum_{k \geq \gamma+2} \mathscr{J}_{j}^{k}\left[G_{\gamma+1}\right]\right)
\end{aligned}
$$


The Johnston index has the property that for $k>\gamma \mathscr{J}_{i}^{k}\left[G_{\gamma}\right]=\mathscr{J}_{i}^{k}\left[G_{\gamma+1}\right]$ thus it is enough to show that when we increase the quorum from $\gamma$ to $\gamma+1$ then

$$
\mathscr{J}_{i}^{k}\left[G_{\gamma}\right]-\mathscr{J}_{j}^{k}\left[G_{\gamma}\right] \geq \mathscr{J}_{i}^{k}\left[G_{\gamma+1}\right]-\mathscr{J}_{j}^{k}\left[G_{\gamma+1}\right], \text { for } k=\gamma, \gamma+1
$$

It is so for $k=\gamma$, because $\mathscr{J}_{i}^{\gamma}\left[G_{\gamma}\right] \geq \mathscr{J}_{j}^{\gamma}\left[G_{\gamma}\right]$ and $\mathscr{J}_{i}^{\gamma}\left[G_{\gamma+1}\right]=0=\mathscr{J}_{j}^{\gamma}\left[G_{\gamma+1}\right]$ as there is no winning coalition of cardinality $\gamma$ in game $G_{\gamma+1}$. Let us now prove the Eq. 15 for $k=\gamma+1$. As in the proof of egalitarian property for Banzhaf score we will consider the following sets:

$$
\begin{aligned}
& S_{i, j}^{+}(k)=\{S \subseteq N:|S|=k+1, i, j \in S\} \\
& S_{i, j}^{-}(k)=\{S \subseteq N:|S|=k, i, j \notin S\}
\end{aligned}
$$

Let us consider the sets of up-swings $\mathrm{Sw}_{i}^{\uparrow}$ in $S_{i, j}^{-}(k)$ and down-swings $\mathrm{Sw}_{i}^{\downarrow}$ in $S_{i, j}^{+}(k)$. Let us recall the definition of the sets of up-swings and down-swings:

$$
\begin{aligned}
& \operatorname{Sw}_{i}^{\uparrow}=\{S \subseteq N: i \notin S, S \notin \operatorname{Win}(G), S \cup\{i\} \in \operatorname{Win}(G)\} \\
& \operatorname{Sw}_{i}^{\downarrow}=\left\{S \in \operatorname{Win}_{i}(G): S \backslash\{i\} \notin \operatorname{Win}_{i}(G)\right\}
\end{aligned}
$$

In the case of $S_{i, j}^{+}(k)$ we have the following partition of this set:

$$
\begin{aligned}
S_{1}^{\downarrow}[G, i, j] & =\{S \in \operatorname{Lo}(G):|S|=\gamma+1, i, j \in S\}=\operatorname{Lo}(G) \cap S_{i, j}^{+}(\gamma) \\
S_{2}^{\downarrow}[G, i, j] & =\left\{S \in \operatorname{Sw}_{j}^{\downarrow}(G):|S|=\gamma+1, i, j \in S\right\}=\operatorname{Sw}_{j}^{\downarrow} \cap S_{i, j}^{+}(\gamma) \\
S_{3}^{\downarrow}[G, i, j] & =\left\{S \in \operatorname{Sw}_{i}^{\downarrow}(G) \backslash \operatorname{Sw}_{j}^{\downarrow}(G):|S|=\gamma+1, i, j \in S\right\} \\
& =\left(\operatorname{Sw}_{i}^{\downarrow}(G) \backslash \operatorname{Sw}_{j}^{\downarrow}(G)\right) \cap S_{i, j}^{+}(\gamma) \\
S_{4}^{\downarrow}[G, i, j] & =\left\{S \in \operatorname{Win}(G) \backslash \operatorname{Sw}_{i}^{\downarrow}:|S|=\gamma+1, i, j \in S\right\} \\
& =\left(\operatorname{Win}(G) \backslash \operatorname{Sw}_{i}^{\downarrow}\right) \cap S_{i, j}^{+}(\gamma)
\end{aligned}
$$

and we have the partition of $S_{i, j}^{-}(\gamma-1)$ :

$$
\begin{aligned}
& S_{1}^{\uparrow}[G, i, j]=\left\{S \in \operatorname{Lo}(G) \backslash \mathrm{Sw}_{i}^{\uparrow}:|S|=\gamma, i, j \notin S\right\}=\left(\operatorname{Lo}(G) \backslash \mathrm{Sw}_{i}^{\uparrow}\right) \cap S_{i, j}^{-}(\gamma-1) \\
& S_{2}^{\uparrow}[G, i, j]=\left\{S \in \operatorname{Sw}_{i}^{\uparrow} \backslash \operatorname{Sw}_{j}^{\uparrow}(G):|S|=\gamma, i, j \notin S\right\}=\left(\operatorname{Sw}_{i}^{\uparrow} \backslash \operatorname{Sw}_{j}^{\uparrow}\right) \cap S_{i, j}^{-}(\gamma-1) \\
& S_{3}^{\uparrow}[G, i, j]=\left\{S \in \operatorname{Sw}_{j}^{\uparrow}(G):|S|=\gamma, i, j \notin S\right\}=\operatorname{Sw}_{j}^{\uparrow}(G) \cap S_{i, j}^{-}(\gamma-1) \\
& S_{4}^{\uparrow}[G, i, j]=\{S \in \operatorname{Win}(G):|S|=\gamma, i, j \notin S\}=\operatorname{Win}(G) \cap S_{i, j}^{-}(\gamma-1)
\end{aligned}
$$




$$
\mathscr{J}_{i}^{\gamma+1}[G]=\mathscr{J}_{i}^{\gamma+1}\left[G_{\gamma}\right]=\sum_{S \in S_{i, j}^{+}(k) \cap S w_{i}^{\downarrow}} \frac{1}{d_{S}}+\sum_{S \in S_{i, j}^{-}(k) \cap S w_{i}^{\uparrow}} \frac{1}{d_{S \cup\{i\}}}
$$

then we can decompose the sum in respect of the given partition of $S_{i, j}^{+}(k)$ and $S_{i, j}^{-}(k)$ :

$$
\begin{aligned}
\mathscr{J}_{i}^{\gamma+1}[G]=\mathscr{J}_{i}^{\gamma+1}\left[G_{\gamma}\right]= & \underbrace{\sum_{S \in S_{1}^{\downarrow}[G, i, j] \cap S \mathrm{w}_{i}^{\downarrow}} \frac{1}{d_{S}}}_{=0}+\sum_{S \in S_{2}^{\downarrow}[G, i, j] \cap S \mathrm{w}_{i}^{\downarrow}} \frac{1}{d_{S}} \\
& +\sum_{S \in S_{3}^{\downarrow}[G, i, j] \cap S \mathrm{w}_{i}^{\downarrow}} \frac{1}{d_{S}}+\sum_{S \in S_{4}^{\downarrow}[G, i, j] \cap S \mathrm{w}_{i}^{\downarrow}} \frac{1}{d_{S}} \\
& +\underbrace{\sum_{S \in S_{1}^{\uparrow}[G, i, j] \cap S \mathrm{w}_{i}^{\uparrow}} \frac{1}{d_{S \cup\{i\}}}}_{=0}+\sum_{S \in S_{2}^{\uparrow}[G, i, j] \cap S \mathrm{w}_{i}^{\uparrow}} \frac{1}{d_{S \cup\{i\}}} \\
& +\sum_{S \in S_{3}^{\uparrow}[G, i, j] \cap S \mathrm{w}_{i}^{\uparrow}} \frac{1}{d_{S \cup\{i\}}}+\sum_{S \in S_{4}^{\uparrow}[G, i, j] \cap S \mathrm{w}_{i}^{\uparrow}} \frac{1}{d_{S \cup\{i\}}}
\end{aligned}
$$

In the game $G$ for the player $i$ :

$$
\begin{aligned}
& S_{1}^{\downarrow}[G, i, j] \cap \mathrm{Sw}_{i}^{\downarrow}=\emptyset \quad S_{1}^{\uparrow}[G, i, j] \cap \mathrm{Sw}_{i}^{\uparrow}=\emptyset \\
& S_{2}^{\downarrow}[G, i, j] \cap \mathrm{Sw}_{i}^{\downarrow}=S_{2}^{\downarrow}[G, i, j] \quad S_{2}^{\uparrow}[G, i, j] \cap \mathrm{Sw}_{i}^{\uparrow}=S_{2}^{\uparrow}[G, i, j] \\
& S_{3}^{\downarrow}[G, i, j] \cap \mathrm{Sw}_{i}^{\downarrow}=S_{3}^{\downarrow}[G, i, j] \quad S_{3}^{\uparrow}[G, i, j] \cap \mathrm{Sw}_{i}^{\uparrow}=S_{3}^{\uparrow}[G, i, j] \\
& S_{4}^{\downarrow}[G, i, j] \cap \mathrm{Sw}_{i}^{\downarrow}=\emptyset \quad S_{4}^{\uparrow}[G, i, j] \cap \mathrm{Sw}_{i}^{\uparrow}=\emptyset
\end{aligned}
$$

And for the player $j$ :

$$
\begin{aligned}
& S_{1}^{\downarrow}[G, i, j] \cap \mathrm{Sw}_{j}^{\downarrow}=\emptyset \quad S_{1}^{\uparrow}[G, i, j] \cap \mathrm{Sw}_{j}^{\uparrow}=\emptyset \\
& S_{2}^{\downarrow}[G, i, j] \cap \mathrm{Sw}_{j}^{\downarrow}=S_{2}^{\downarrow}[G, i, j] \quad S_{2}^{\uparrow}[G, i, j] \cap \mathrm{Sw}_{j}^{\uparrow}=\emptyset \\
& S_{3}^{\downarrow}[G, i, j] \cap \mathrm{Sw}_{j}^{\downarrow}=\emptyset \quad S_{3}^{\uparrow}[G, i, j] \cap \mathrm{Sw}_{j}^{\uparrow}=S_{3}^{\uparrow}[G, i, j] \\
& S_{4}^{\downarrow}[G, i, j] \cap \mathrm{Sw}_{j}^{\downarrow}=\emptyset \quad S_{4}^{\uparrow}[G, i, j] \cap \mathrm{Sw}_{j}^{\uparrow}=\emptyset
\end{aligned}
$$


Then in the games $G$ and $G_{\gamma}$ we get:

$$
\begin{aligned}
\mathscr{J}_{i}^{\gamma+1}[G]=\mathscr{J}_{i}^{\gamma+1}\left[G_{\gamma}\right]= & \sum_{S \in S_{2}^{\downarrow}[G, i, j]} \frac{1}{d_{S}}+\sum_{S \in S_{3}^{\downarrow}[G, i, j]} \frac{1}{d_{S}} \\
& +\sum_{S \in S_{2}^{\uparrow}[G, i, j]} \frac{1}{d_{S \cup\{i\}}}+\sum_{S \in S_{3}^{\uparrow}[G, i, j]} \frac{1}{d_{S \cup\{i\}}} \\
\mathscr{J}_{j}^{\gamma+1}[G]=\mathscr{J}_{j}^{\gamma+1}\left[G_{\gamma}\right]= & \sum_{S \in S_{2}^{\downarrow}[G, i, j]} \frac{1}{d_{S}}+\sum_{S \in S_{3}^{\uparrow}[G, i, j]} \frac{1}{d_{S \cup\{j\}}}
\end{aligned}
$$

thus their difference is:

$$
\begin{aligned}
\mathscr{J}_{i}^{\gamma+1}[G]-\mathscr{J}_{j}^{\gamma+1}[G]= & \sum_{S \in S_{2}^{\uparrow}[G, i, j]} \frac{1}{d_{S \cup\{j\}}}+\sum_{S \in S_{3}^{\downarrow}[G, i, j]} \frac{1}{d_{S}} \\
& +\sum_{S \in S_{3}^{\uparrow}[G, i, j]} \frac{1}{d_{S \cup\{i\}}}-\sum_{S \in S_{3}^{\uparrow}[G, i, j]} \frac{1}{d_{S \cup\{j\}}} \\
\geq & \underbrace{\left(\left|S_{2}^{\uparrow}[G, i, j]\right|+\left|S_{3}^{\downarrow}[G, i, j]\right|\right) \frac{1}{\gamma+1}}_{=: A}
\end{aligned}
$$

and in the game $G_{\gamma+1}$ :

$$
\begin{aligned}
& \mathscr{J}_{i}^{\gamma+1}\left[G_{\gamma+1}\right] \\
& \sum_{S \in S_{2}^{\downarrow}[G, i, j]} \frac{1}{d_{S}\left[G_{\gamma+1}\right]}+\sum_{S \in S_{3}^{\downarrow}[G, i, j]} \frac{1}{d_{S}\left[G_{\gamma+1}\right]} \\
& \left.\begin{array}{rl}
= & +\sum_{S \in S_{2}^{\uparrow}[G, i, j]} \frac{1}{d_{S \cup\{i\}}\left[G_{\gamma+1}\right]}+\sum_{S \in S_{3}^{\uparrow}[G, i, j]} \frac{1}{d_{S \cup\{i\}}\left[G_{\gamma+1}\right]} \\
+\sum_{S \in S_{4}^{\uparrow}[G, i, j]} \frac{1}{d_{S \cup\{i\}}\left[G_{\gamma+1}\right]}+\sum_{S \in S_{4}^{\downarrow}[G, i, j]} \frac{1}{d_{S}\left[G_{\gamma+1}\right]}
\end{array}\right\} \leq \mathscr{J}_{i}^{\gamma+1}\left[G_{\gamma}\right] \\
& =\left(\left|S_{2}^{\downarrow}[G, i, j]\right|+\left|S_{3}^{\downarrow}[G, i, j]\right|+\left|S_{2}^{\uparrow}[G, i, j]\right|+\left|S_{3}^{\uparrow}[G, i, j]\right|\right. \\
& \left.+\left|S_{4}^{\uparrow}[G, i, j]\right|+\left|S_{4}^{\downarrow}[G, i, j]\right|\right) \frac{1}{\gamma+1}
\end{aligned}
$$




$$
\begin{aligned}
\mathscr{J}_{j}^{\gamma+1}\left[G_{\gamma+1}\right]= & \underbrace{}_{\leq \mathscr{J}_{j}^{\gamma+1}\left[G_{\gamma}\right]} \frac{1}{d_{S}\left[G_{\gamma+1}\right]}+\sum_{S \in S_{3}^{\uparrow}[G, i, j]} \frac{1}{d_{S \cup\{j\}}\left[G_{\gamma+1}\right]} \\
& +\sum_{S \in S_{3}^{\downarrow}[G, i, j]} \frac{1}{d_{S}\left[G_{\gamma+1}\right]}+\sum_{S \in S_{4}^{\uparrow}[G, i, j]} \frac{1}{d_{S \cup\{j\}}\left[G_{\gamma+1}\right]}+\sum_{S \in S_{4}^{\downarrow}[G, i, j]} \frac{1}{d_{S}\left[G_{\gamma+1}\right]} \\
= & \left(\left|S_{2}^{\downarrow}[G, i, j]\right|+\left|S_{3}^{\uparrow}[G, i, j]\right|+\left|S_{3}^{\downarrow}[G, i, j]\right|+\left|S_{4}^{\uparrow}[G, i, j]\right|+\left|S_{4}^{\downarrow}[G, i, j]\right|\right) \\
& \times \frac{1}{\gamma+1}
\end{aligned}
$$

thus the difference is:

$$
\mathscr{J}_{i}^{\gamma+1}\left[G_{\gamma+1}\right]-\mathscr{J}_{j}^{\gamma+1}\left[G_{\gamma+1}\right]=\left|S_{2}^{\uparrow}[G, i, j]\right| \frac{1}{\gamma+1} \leq A
$$

where $A$ was defined in formula (16). What ends the proof.

\section{Proof of Remark 5}

Counter Example 4 Let $G=[8 ; 3,2,2,1,1,1]$. The player with weight 3 will be denoted as 1 , players with weight 2 as $2_{1}, 2_{2}$ and players with weight 1 as $3_{1}, 3_{2}, 3_{3}$. The Banzhaf score and (normalized) index are as follows:

\begin{tabular}{cccc|ccc} 
& \multicolumn{3}{c}{ Banzhaf score } & \multicolumn{3}{c}{ Banzhaf index } \\
game $\backslash$ player: & 1 & $2_{1}$ & $3_{1}$ & 1 & $2_{1}$ & $3_{1}$ \\
$G$ & 9 & 7 & 3 & 0.28 & 0.22 & 0.09 \\
$G \cap Q_{5}$ & 6 & 4 & 4 & 0.23 & 0.15 & 0.15
\end{tabular}

and the Johnston score and index:

\begin{tabular}{cccc|ccc} 
& \multicolumn{3}{c}{ Johnston score } & \multicolumn{3}{c}{ Johnston index } \\
game player: & 1 & 2 & $3_{1}$ & 1 & $2_{1}$ & $3_{1}$ \\
$G$ & 5 & 4 & 3 & 0.23 & 0.18 & 0.13 \\
$G \cap Q_{5}$ & 5 & 4 & 4 & 0.25 & 0.16 & 0.16
\end{tabular}

It is important to observe that the difference of the Banzhaf index between players 1 and $2_{1}$ are growing when we pass from $G$ to $G \cap Q_{5}$ : from $\frac{2}{32} \simeq 0.06$ to $\frac{2}{26} \simeq 0.08$. Similarly for the Johnston index: the difference in $G$ is 0.05 and in $G \cap Q_{5}$ it is equal to 0.09 .

\section{Proof of Remark 6}

Holler and Deegan-Packel indices in contrast with the previously considered indices do not respect the desirability order. Let us consider the orders given by values of Holler index $(\chi)$ or Deegan-Packel index $(\delta)$. We will denote these orders by $\succsim_{\chi}$ and $\succsim_{\delta}$ respectively, i.e., $i \succsim_{\chi} j$ in a game $G$ if and only if $\chi_{i}[G] \geq \chi_{j}[G]$ and similarly 
for $\succsim \delta$. In the example below, we show that the orders can be contradictory when we consider the set of orders coming from an egalitarian sequence of games, i.e., it can happen that $i>_{\chi} j$ in $G \cap Q_{\gamma_{1}}$ and $i<_{\chi} j$ in $G \cap Q_{\gamma_{2}}$ for $\gamma_{1}<\gamma_{2}$.

Counter Example 5 Let us consider a weighted simple game: $G=[5 ; 5,1,1,1,1,1$, $1,1,1,1,1,0]$. Player 1 is the one with weight 5 , players with weight 1 will be denoted as $2_{1}, 2_{2}, \cdots, 2_{10}$ and player 3 is the null player. The Deegan-Packel score and the Deegan-Packel index in $G$ and $G_{5}$ are respectively equal to:

\begin{tabular}{cccc|ccc} 
& \multicolumn{3}{c}{ Deegan-Packel score } & \multicolumn{3}{c}{ Deegan-Packel index } \\
game $\backslash$ player: & 1 & 21 & 3 & 1 & 21 & 3 \\
$G$ & 1 & 25.2 & 0 & 0.004 & 0.1 & 0 \\
$G \cap Q_{5}$ & 66 & 49.2 & 24 & 0.11 & 0.08 & 0.04
\end{tabular}

and the Holler score and the Holler index are respectively:

\begin{tabular}{cccc|ccc} 
& \multicolumn{3}{c}{ Holler score } & \multicolumn{3}{c}{ Holler index } \\
game $\backslash$ player: & 1 & 21 & 3 & 1 & $2_{1}$ & 3 \\
$G$ & 1 & 1.26 & 0 & 0.001 & 0.01 & 0 \\
$G \cap Q_{5}$ & 330 & 246 & 120 & 0.11 & 0.085 & 24
\end{tabular}

We can observe that when passing from $G$ to $G \cap Q_{5}$ the power of a stronger player is growing more than the power of a smaller player for the four evaluations, so that all these four indices from the above tables do not satisfy the egalitarian property.

In the above examples both indices: Holler and Deegan-Packel give more value to a weaker player than to a stronger player with respect to the desirability relation in the game $G$ but in the game $G \cap Q_{5}$, after intersecting it with the symmetric game with consensus level 5, the stronger player gains more power according to these indices and the orders $\succsim_{\chi}$ and $\succsim_{\delta}$ are now reversed. Egalitarian property for an index means that weaker players with respect to the desirability relation gain more value after adding a stronger quorum condition, which is not the case of these indices.

\section{E Proof of Theorem 3}

Let $\psi$ be an efficient power index on $S_{n}$ fulfilling the egalitarian property and respecting the desirability relation, i.e., $\psi_{1} \geq \psi_{2} \geq \cdots \geq \psi_{n}$ for any simple game $v \in S_{n}$. For complete simple games, egalitarian property implies in particular the following:

$$
\psi_{1}\left[v_{1}\right]-\psi_{n}\left[v_{1}\right] \geq \psi_{1}\left[v_{2}\right]-\psi_{n}\left[v_{2}\right]
$$

where $v_{1}=u \cap Q_{\gamma_{1}}, v_{2}=u \cap Q_{\gamma_{2}}, 1 \leq \gamma_{1}<\gamma_{2} \leq n$, and $u$ is a complete simple game (or a simple game such that $v_{1}$ is complete). Then efficiency of $\psi$ implies that $\sum_{i \in N} \psi_{i}\left[v_{1}\right]=\sum_{i \in N} \psi_{i}\left[v_{2}\right]=1$.

First, we will show that $\psi_{n}\left[v_{2}\right] \geq \psi_{n}\left[v_{1}\right]$ and $\psi_{1}\left[v_{2}\right] \leq \psi_{1}\left[v_{1}\right]$. Ad absurdum: let us assume that $\epsilon=\psi_{n}\left[v_{1}\right]-\psi_{n}\left[v_{2}\right]>0$. Then by the egalitarian property $\psi_{i}\left[v_{1}\right]-\psi_{n}\left[v_{1}\right] \geq \psi_{i}\left[v_{2}\right]-\psi_{n}\left[v_{2}\right]$ if and only if $\psi_{i}\left[v_{1}\right]-\psi_{i}\left[v_{2}\right] \geq \psi_{n}\left[v_{1}\right]-\psi_{n}\left[v_{2}\right]=$ 
$\epsilon>0$ and taking the sum for all players:

$$
\sum_{i \in N} \psi_{i}\left[v_{1}\right]-\sum_{i \in N} \psi_{i}\left[v_{2}\right] \geq n \epsilon>0
$$

a contradiction with the efficiency of $\psi$.

A similar argument shows that the value of the strongest player must decrease when passing from $v_{1}$ to $v_{2}$.

Both functions: $N \ni i \mapsto \psi_{i}\left[v_{k}\right], k=1,2$ are monotonic and $\psi$ is egalitarian thus the above property implies that there exists player $i_{0}$ such that $\psi_{i}\left[v_{2}\right]>\psi_{i}\left[v_{1}\right]$ for $i>i_{0}$ and $\psi_{i}\left[v_{2}\right] \leq \psi_{i}\left[v_{1}\right]$ for $i \leq i_{0}$. If such $i_{0}$ does not exist then the egalitarian property of $\psi$ would not hold.

For $k \geq i_{0}$ we have $\sum_{i \geq k} \psi_{i}\left[v_{2}\right] \geq \sum_{i \geq k} \psi_{i}\left[v_{1}\right]$. It remains to consider the case $k<i_{0}$. Then

$$
\begin{aligned}
\sum_{i \leq k} \psi_{i}\left[v_{2}\right]-\sum_{i \leq k} \psi_{i}\left[v_{1}\right] & =(\underbrace{\sum_{i_{0} \leq i \leq k} \psi_{i}\left[v_{2}\right]-\sum_{i_{0} \leq i \leq k} \psi_{i}\left[v_{1}\right]}_{\leq 0})+(\underbrace{\sum_{i<i_{0}} \psi_{i}\left[v_{2}\right]-\sum_{i<i_{0}} \psi_{i}\left[v_{1}\right]}_{\geq 0}) \\
& \geq\left(\sum_{i_{0} \leq i} \psi_{i}\left[v_{2}\right]-\sum_{i_{0} \leq i} \psi_{i}\left[v_{1}\right]\right)+\left(\sum_{i<i_{0}} \psi_{i}\left[v_{2}\right]-\sum_{i<i_{0}} \psi_{i}\left[v_{1}\right]\right)=0
\end{aligned}
$$

The last equality follows from efficiency of $\psi$, thus the left-hand side of the above equation is greater than or equal to 0 and so the theorem holds.

\section{References}

Alonso-Meijide J, Freixas J (2010) A new power index based on minimal winning coalitions without any surplus. Decis Support Syst 49:70-76

Banzhaf J (1965) Weighted voting doesn't work: a mathematical analysis. Rutgers Law Rev 19:317-343

Carreras F, Freixas J (2004) A power analysis of linear games with consensus. Math Soc Sci 48:207-221

Carreras F, Freixas J (2008) On ordinal equivalence of power measures given by regular semivalues. Math Soc Sci 55:221-234

Carreras F, Freixas J, Puente M (2003) Semivalues as power indices. Eur J Oper Res 149:676-687

Coleman J (1971) Control of collectivities and the power of a collectivity to act. In: Lieberman B, Falchikov N (eds) Social Choice. Gordon \& Breach, New York pp 269-300

Deegan J, Packel E (1978) A new index of power for simple $n$-person games. Int J Game Theory 7:113-123

Felsenthal D, Machover M (1998) The measurement of voting power. Edward Elgar Publishing Limited, Cheltenham

Felsenthal D, Machover M (2001) The Treaty of Nice and qualified majority voting. Soc Choice Welf 18:431-464

Freixas J (2010) On ordinal equivalence of the Shapley and Banzhaf values for cooperative games. Int J Game Theory 39:513-527

Gambarelli G, Owen G (2002) Power in political and business structures. In: Holler M, Kliemt H, Schmidtchen D, Streit M (eds) Power and fairness, Jahrbuch für Neue Politische Ökonomie, vol 20. Tübingen, Mohr-Siebeck, pp 57-68

Hirokawa M, Vlach M (2006) Power analysis of voting by count and account. Kybernetika 42:483-493

Hirokawa M, Xu P (2005) Small creditors' power in civil rehablitation-a compound game of a simple majority and a weighted majority. Technical Report, Hosei University, Mimeo.

Holler M (1982) Forming coalitions and measuring voting power. Political Stud 30:262-271 
Holler M (2002) On power and fairness and their relationships: an introductory chapter. In: Holler M, Kliemt H, Schmidtchen D, Streit M (eds) Power and fairness, Jahrbuch für Neue Politische Ökonomie, vol 20. Tübingen, Mohr-Siebeck, pp 1-27

Isbell J (1958) A class of simple games. Duke Math J 25:423-439

Johnston R (1978) On the measurement of power: some reactions to Laver. Environ Plan A 10:907-914

Laruelle A, Valenciano F (2002) Inequality among EU citizens in the EU's council decision procedure. Eur J Political Econ 18:475-498

Peleg B (1992) Voting by count and account. In: Selten R (ed) Rational interaction. Springer-Verlag, Berlin, pp 307-317

Rae D (1969) Decision-rules and individual values in constitutional choice. Am Political Sci Rev 63:40-56

Shapley L, Shubik M (1954) A method for evaluating the distribution of power in a committee system. Am Political Sci Rev 48:787-792

Taylor A (1995) Mathematics and politics. Strategy, voting, power and proof. Textbooks in mathematical sciences. Springer-Verlag, New York

Taylor A, Zwicker W (1999) Simple games. Desirability relations, trading, pseudoweightings. Princeton University Press, Princeton, NJ

Turnovec F (2002) Evaluation of Commission's proposal of double simple majority voting rule in the Council of Ministers of the European Union. In: Holler M, Kliemt H, Schmidtchen D, Streit M (eds) Power and fairness, Jahrbuch fur Neue Politische Okonomie, vol 20. Mohr Siebeck, Tufbingen, pp 103-122

Weymark J (1981) Generalized Gini inequality indices. Math Soc Sci 1:409-430 